\title{
Splenic extramedullary hematopoietic proliferation in Philadelphia chromosome-negative myeloproliferative neoplasms: heterogeneous morphology and cytological composition
}

\author{
Sonam Prakash ${ }^{1}$, Ronald Hoffman ${ }^{2}$, Sharon Barouk ${ }^{1}$, Y Lynn Wang ${ }^{1}$, Daniel M Knowles ${ }^{1}$ \\ and Attilio Orazi ${ }^{1}$ \\ ${ }^{1}$ Department of Pathology and Laboratory Medicine, Weill Cornell Medical College, New York, NY, USA and \\ ${ }^{2}$ Tisch Cancer Institute, Mount Sinai School of Medicine, New York, NY, USA
}

\begin{abstract}
We studied 24 spleens with extramedullary hematopoietic proliferation (EMHP), a key feature of advanced-stage Philadelphia chromosome-negative myeloproliferative neoplasms, obtained from 24 patients (14 primary myelofibrosis, 7 polycythemia vera and 3 unclassifiable). Hematoxylin and eosin, reticulin and trichrome stains, and immunohistochemical stains for myeloperoxidase, glycophorin-C, CD42b, CD34, CD117, CD8, nerve growth factor receptor and smooth muscle actin were evaluated. Clinical information was correlated with the morphological findings. Three distinct histological patterns of EMHP were recognized: diffuse (12), nodular (5), and mixed-nodular and diffuse (7). The preponderant lineage was granulocytic in diffuse, trilineage in nodular and erythroid in mixed EMHP. Erythropoiesis was largely intravascular, granulopoiesis was within the splenic cords and megakaryopoiesis was observed in both locations. The stromal changes paralleled the histological pattern with preservation of the splenic stromal and vascular architecture in the diffuse areas as opposed to areas of nodular EMHP. The morphological features of the splenic EMHP did not correlate with specific subtypes of myeloproliferative neoplasms. The mean duration of follow-up from initial diagnosis was $\mathbf{8 0}$ months. A total of 15 of the 24 patients died of disease: 8 of $12(67 \%)$ with diffuse, 2 of $5(40 \%)$ with nodular and 5 of $7(71 \%)$ with mixed growth patterns. The mean duration from diagnosis to splenectomy was shorter in patients with diffuse ( 83 months) as compared with those with nodular EMHP (127 months). Our study demonstrates that splenic extramedullary hematopoietic proliferation in Philadelphia chromosome-negative myeloproliferative neoplasms shows distinct histological patterns that do not correlate with disease subtypes, but appear to suggest a trend between the histological patterns and clinical behavior. These results suggest a different biology of the disease in the nodular and diffuse extramedullary hematopoietic proliferation groups. Modern Pathology (2012) 25, 815-827; doi:10.1038/modpathol.2012.33; published online 2 March 2012
\end{abstract}

Keywords: extramedullary hematopoiesis; myeloproliferative neoplasms; spleen

Extramedullary hematopoiesis resulting in progressive splenomegaly is a key feature of advanced-stage disease in Philadelphia chromosome-negative myeloproliferative neoplasms, a group of hematological

Correspondence: Dr A Orazi, MD, Department of Pathology and Laboratory Medicine, Weill Cornell Medical College, 525 East 68th Street, Room ST-707, Starr 715, New York, NY 10065, USA. E-mail: ato9002@med.cornell.edu

Received 5 August 2011; revised 4 January 2012; accepted 4 January 2012; published online 2 March 2012 neoplasms that includes essential thrombocythemia, polycythemia vera, primary myelofibrosis and myeloproliferative neoplasm-unclassifiable. ${ }^{1,2}$ The occurrence of extramedullary hematopoiesis in myeloproliferative neoplasms is associated with abnormal trafficking patterns of clonal hematopoietic progenitor cells and hematopoietic stem cells attributed to dysregulation of the bone marrow microenvironment. This results in circulating mature and immature marrow elements that home to the spleen. ${ }^{3-5}$ There, the bone marrow-derived cells 
undergo sustained malignant multilineage hematopoiesis, resulting in progressive splenomegaly. Studies based on the identification of loss of heterozygosity ${ }^{6}$ and presence of $J A K 2^{V 617}$ mutation $^{7,8}$ have provided evidence that splenic extramedullary hematopoiesis in myeloproliferative neoplasms is clonal, supporting the hypothesis that hematopoietic cells causing the splenic proliferation are derived from the transformed bone marrow clones. To further emphasize the neoplastic nature of splenic extramedullary hematopoiesis in myeloproliferative neoplasms, and to distinguish it from reactive extramedullary hematopoiesis, O'Malley et $a l^{9}$ have proposed to use the term, 'splenic hematopoietic proliferation,' in this disease context.

It is well known that the spleen is a major site of disease as well as disease progression in the advanced stages of Philadelphia chromosome-negative myeloproliferative neoplasms, and that acute leukemic transformation in these neoplasms can occur in the spleen. ${ }^{10}$ Nonetheless, there is very limited information on the pathogenetic mechanisms involved in this phenomenon. Most of the studies elucidating the biology of Philadelphia chromosomenegative myeloproliferative neoplasms have been performed on bone marrow-derived or circulating hematopoietic stem cells/hematopoietic progenitor cells. In contrast with the extensive available literature on the histological findings of myeloproliferative neoplasms in the bone marrow, the morphological and immunohistochemical features of the splenic extramedullary hematopoietic proliferation (EMHP) in these neoplasms have not been extensively studied. A previous study by Mesa et $a l^{11}$ evaluated the prognostic value of splenic histopathology in myelofibrosis with myeloid metaplasia, based on microscopic examination of hematoxylin and eosin-stained sections of spleen. O'Malley et $a l^{9}$ evaluated the differential morphological and immunohistochemical features of the splenic hematopoietic proliferations in neoplastic and benign disorders. The aim of the current study was to evaluate the histological and immunohistochemical features of EMHP in the spleen from patients with Philadelphia chromosome-negative myeloproliferative neoplasms and to see if they correlate with the specific subtypes, as well as clinical course.

\section{Materials and methods}

The files of the Department of Pathology and Laboratory Medicine of the Weill Cornell Medical Center/New York Presbyterian Hospital (New York, USA) were searched for splenectomy specimens from patients previously diagnosed with Philadelphia chromosome-negative myeloproliferative neoplasms, and which contained EMHP. Information concerning gross pathology on each case was obtained from the pathology records. Representative formalin-fixed, paraffin-embedded tissue blocks were selected for microscopic evaluation. Hematoxylin and eosin-stained sections were utilized to evaluate the splenic morphology. The histological growth pattern of EMHP was classified as diffuse, nodular, or mixed-nodular and diffuse. The classification of cases into diffuse EMHP required the presence of $>75 \%$ diffuse pattern, and nodular EMHP required the presence of $>75 \%$ nodular pattern. Cases without an obvious predominance of nodular or diffuse pattern were classified as mixed-nodular and diffuse EMHP. The cellular composition and spatial localization of each hematopoietic cell lineage was studied. The proportion of each hematopoietic lineage was graded semiquantitatively as $1+(<25 \%$ of total EMHP), $2+(25-50 \%$ of total EMHP) or $3+(>50 \%$ of total EMHP). The localization of hematopoietic cells in microvessels, splenic sinusoids or splenic cords was evaluated.

Immunohistochemical stains were performed using an automated immunostainer (Bond Max, Leica Microsystems, IL, USA). The manufacturers and staining information are listed in Table 1. Immunohistochemical stains for myeloperoxidase, glycophorin-C and $\mathrm{CD} 42 \mathrm{~b}$ were performed to analyze cells of the granulocytic, erythroid and megakaryocytic lineages, respectively. Additionally, immunohistochemistry for CD34, CD8, nerve growth factor receptor (NGFR) and smooth muscle actin (SMA) was performed to evaluate the stromal components, that is, microvessels, splenic sinusoids, adventitial reticulum cells and pericytes, respectively. Immunohistochemistry for CD34 and CD117 was performed to evaluate the presence of immature hematopoietic cells. Reticulin and trichrome stains were utilized to evaluate the extent of

Table 1 Immunohistochemical stains used: source, clone and dilution

\begin{tabular}{llrl}
\hline Immunohistochemical stain & Clone & Dilution & Source \\
\hline Myeloperoxidase & Polyclonal & $1: 5000$ & Dako, Carpenteria, CA, USA \\
Glycophorin C & Ret40f & $1: 50$ & Abcam, Cambridge, MA, USA \\
CD42b & MM2/174 & Novocastra, Newcastle upon Tyne, UK \\
CD34 & QBEnd/10 & $1: 50$ & Biogenex, San Ramon, CA, USA \\
CD117 & Polyclonal & $1: 50$ & Dako, Carpenteria, CA, USA \\
CD8 & C8/144B & $1: 75$ & Dako, Carpenteria, CA, USA \\
NGFR & ME 20.4 & $1: 75$ & Abcam, Cambridge, MA, USA \\
SMA & 1A4 & $1: 1600$ & Dako, Carpenteria, CA, USA
\end{tabular}

Abbreviations: NGFR, nerve growth factor receptor; SMA, smooth muscle actin. 
fibrosis. To assess the degree of stromal alteration, the stromal elements were compared with those seen in three incidental splenectomy specimens secondary to trauma without an underlying splenic pathology.

$J A K 2^{V 617 F}$ mutational analysis was performed on DNA extracted from formalin-fixed, paraffinembedded tissue sections from the spleens, using QIAamp DNA Mini Kit (Qiagen, Valencia, CA, USA). A multiplex PCR assay based on amplification refractory mutation system technology was performed as described previously. ${ }^{12}$ This method detects $J A K 2^{V 617 F}$ allele at a sensitivity of $0.1 \%$.

Correlation between the growth pattern of EMHP, cellular composition and stromal alterations was evaluated. Additionally, clinical information was obtained from the patient records and correlated with the pathological findings.

This study was approved by the Institutional Review Board of Weill Cornell Medical College.

\section{Results}

The main clinical and morphological findings are summarized in Table 2. Twenty-four splenectomy specimens from patients previously diagnosed with Philadelphia chromosome-negative myeloproliferative neoplasms were retrieved over a 14-year period. Where available, the original diagnostic reports and/ or slides, as well as clinical notes, were reviewed to confirm the initial diagnosis according to the current 2008 World Health Organization classification of chronic myeloproliferative neoplasms. ${ }^{13} \mathrm{~A}$ total of 14 of these 24 patients were diagnosed with primary myelofibrosis, and 7 were diagnosed with polycythemia vera. For three cases, the original diagnostic pathology slides or reports, as well as adequate clinical information for further subclassification of the myeloproliferative neoplasm, were not available and were, therefore, diagnosed as myeloproliferative neoplasm not further classified. The patients ranged in age from 43 to 81 years (median 61 years) and included 15 males and 9 females (male to female ratio 1.7:1). The indications for splenectomy included symptoms due to large size, splenic infarction, intra-abdominal bleeding and increasing transfusion requirement due to splenic sequestration. The time from diagnosis to symptomatic splenomegaly ranged from 0 to 258 months (mean 88.7 months, median 46 months).

\section{Histopathological Features}

The weight of the spleens ranged from 965 to $7650 \mathrm{~g}$ (mean $3450 \mathrm{~g}$, median $2691 \mathrm{~g}$ ). The splenic weight did not correlate with the subtype of the myeloproliferative neoplasm. On gross examination, discrete nodular lesions were identified in only 2 of these 24 spleens (case 21 and case 23). Morphological examination of the spleens revealed three distinct histological growth patterns of EMHP: diffuse, nodular, and mixed-nodular and diffuse EMHP (Figure 1). Diffuse EMHP was seen in 12 cases, nodular EMHP in 5 cases, and mixed-nodular and diffuse EMHP in 7 cases. Those cases classified as diffuse EMHP showed hematopoietic proliferation in the red pulp in a predominantly diffuse growth pattern comprising greater than $75 \%$ of the total hematopoietic proliferation. Those cases classified as nodular EMHP showed well-defined nodules of hematopoietic proliferation comprising greater than $75 \%$ of the total splenic hematopoietic proliferation. Two of the five cases of nodular EMHP met the criteria for sclerosing extramedullary myeloid tumor. ${ }^{14}$ One of these cases was associated with primary myelofibrosis, and in the other case, the myeloproliferative neoplasm could not be further sub-classified. Cases of mixed-nodular and diffuse EMHP showed variable proportions of diffuse and nodular areas of hematopoietic proliferation. The extent of the diffuse component of EMHP ranged from 40 to $60 \%$ (mean $47 \%$, median $40 \%$ ) in the cases of mixed EMHP. In these cases, the nodules were much smaller in size as compared with the well-defined larger nodules seen in cases of nodular EMHP. Although megakaryocytes were readily identifiable with hematoxylin and eosin staining, the erythroid and granulocytic precursors were better identified with glycophorin-C and myeloperoxidase immunohistochemical stains, respectively. The nodules of EMHP showed either panmyelosis or a predominance of erythroid precursors. Areas with a diffuse growth pattern of EMHP showed predominantly granulocytic precursors. The different hematopoietic cell lineages showed preferential spatial localization within the diffuse areas. Erythroid precursors were present predominantly within the splenic sinusoids, the granulocytic precursors were mostly within the splenic cords and megakaryocytes were observed in both sites (Figure 2). Nine cases showed greater than $20 \%$ immature precursors, recognizable morphologically by the presence of large nuclei containing conspicuous nucleoli. The percentage of immature cells in these 9 cases ranged from 20 to $80 \%$ (mean $32 \%$, median $20 \%$ ). Three of these cases showed greater than $50 \%$ immature precursors and were diagnosed as compatible with extramedullary acute leukemic transformation (Figure 3). Although this acute leukemic transformation is biologically related to myeloid sarcoma, the clinical context and morphological features, including the absence of a discrete tumor mass composed of immature cells, do not meet the criteria for a diagnosis of myeloid sarcoma as stated in the 2008 World Health Organization classification of tumors of hematopoietic and lymphoid tissues. ${ }^{13}$ Peripheral blood samples at the time of splenectomy were available in all three cases. Bone marrow samples within 40 days before splenectomy were available in two of these three cases. In one case, the peripheral 
Table 2 Summary of histopathological and clinical features of splenic EMHP in Ph-MPN cases

\begin{tabular}{|c|c|c|c|c|c|c|c|c|c|c|c|}
\hline \multirow{2}{*}{$\begin{array}{l}\text { Case } \\
\text { number }\end{array}$} & \multirow{2}{*}{$\begin{array}{c}\text { Original } \\
\text { diagnosis }\end{array}$} & \multirow{2}{*}{$\begin{array}{l}\text { Age/ } \\
\text { sex }\end{array}$} & \multirow{2}{*}{$\begin{array}{l}\text { Histological } \\
\text { growth pattern } \\
\text { of EMHP }\end{array}$} & \multirow{2}{*}{$\begin{array}{c}\text { Spleen } \\
\text { weight (g) }\end{array}$} & \multicolumn{3}{|c|}{ Hematopoietic lineage of EMHP } & \multirow{2}{*}{$\begin{array}{c}\text { Transformation } \\
\text { to } A M L\end{array}$} & \multirow{2}{*}{$\begin{array}{l}\text { Duration from diagnosis } \\
\text { to splenectomy } \\
\text { (months) }\end{array}$} & \multirow{2}{*}{$\begin{array}{l}\text { Duration of follow-up } \\
\text { from splenectomy } \\
\text { (months) }\end{array}$} & \multirow[t]{2}{*}{ Outcome } \\
\hline & & & & & Granulocytic & Erythroid & Megakaryocytic & & & & \\
\hline 1 & PV & $81 / \mathrm{m}$ & D & 2700 & $3+(\mathrm{IM})$ & $2+$ & $1+$ & + & 36 & 5 & DOD \\
\hline 2 & PV & $57 / \mathrm{m}$ & D & 3720 & $2+(\mathrm{IM})$ & $2+$ & $2+$ & + & 213 & 15 & DOD \\
\hline 3 & MPN-U & $64 / \mathrm{m}$ & $\mathrm{D}$ & 7640 & $3+$ & $1+$ & $2+$ & - & NA & 1.5 & DOD \\
\hline 4 & PMF & $43 / \mathrm{m}$ & $\mathrm{D}$ & 2000 & $2+$ & $2+$ & $2+$ & - & 12 & 38 & DOD \\
\hline 5 & PV & $73 / \mathrm{m}$ & $\mathrm{D}$ & 2682 & $2+$ & $3+(\mathrm{IM})$ & $1+$ & - & 10 & 12 & DOD \\
\hline 6 & PMF & $74 / \mathrm{f}$ & $\mathrm{D}$ & 2450 & $2+$ & $2+$ & $2+$ & - & NA & 16 & DOD \\
\hline 7 & PMF & $61 / \mathrm{m}$ & D & 2535 & $1+$ & $3+(\mathrm{IM})$ & $1+$ & + & NA & 22 & DOD \\
\hline 8 & PMF & $71 / f$ & $\mathrm{D}$ & 1800 & $2+$ & $3+$ & $1+$ & + & 120 & 9 & DOD \\
\hline 9 & PV & $55 / \mathrm{f}$ & D & 5350 & $1+$ & $3+(\mathrm{IM})$ & $1+$ & + & 240 & 3 & AWD \\
\hline 10 & PMF & $79 / \mathrm{f}$ & $\mathrm{D}$ & 1500 & $3+$ & $1+$ & $1+$ & - & 36 & 36 & AWD \\
\hline 11 & PMF & $61 / \mathrm{m}$ & $\mathrm{D}$ & 2500 & $3+$ & $1+$ & $1+$ & - & 24 & 9 & AWD \\
\hline 12 & $\mathrm{PMF}$ & $53 / \mathrm{m}$ & $\mathrm{D}$ & 2000 & $3+$ & $1+$ & $1+$ & - & 56 & 7 & AWD \\
\hline 13 & PMF & $61 / \mathrm{m}$ & $\mathrm{M}$ & 4510 & $1+$ & $3+(\mathrm{IM})$ & $1+$ & - & 26 & 0.5 & DOD \\
\hline 14 & PMF & $62 / \mathrm{m}$ & M & 7650 & $2+(\mathrm{IM})$ & $3+$ & $1+$ & - & 72 & 19 & DOD \\
\hline 15 & MPN-U & $51 / \mathrm{m}$ & M & 6445 & $2+$ & $2+$ & $2+$ & - & NA & 0.01 & DOD \\
\hline 16 & PV & $71 / \mathrm{f}$ & M & 4108 & $3+$ & $2+$ & $1+$ & - & 72 & 84 & DOD \\
\hline 17 & PMF & $71 / \mathrm{m}$ & M & 5400 & $2+$ & $2+$ & $2+$ & - & 21 & 1 & DOD \\
\hline 18 & PV & $61 / f$ & M & 2200 & $1+$ & $3+$ & $2+$ & - & 276 & 36 & AWD \\
\hline 19 & PMF & $58 / \mathrm{f}$ & M & 965 & $3+$ & $1+$ & $2+$ & - & NA & 2 & AWD \\
\hline 20 & PMF & $67 / \mathrm{m}$ & $\mathrm{N}$ & 5290 & $2+$ & $2+$ & $2+$ & - & NA & 2 & DOD \\
\hline 21 & $\mathrm{PMF}^{\mathrm{a}}$ & $60 / \mathrm{m}$ & $\mathrm{N}$ & 1505 & $1+$ & $3+$ & $2+$ & + & 13 & 17 & DOD \\
\hline 22 & PV & $58 / \mathrm{f}$ & $\mathrm{N}$ & 3180 & $2+(\mathrm{IM})$ & $2+$ & $2+$ & + & 258 & 6 & AWD \\
\hline 23 & MPN-U ${ }^{a}$ & $58 / \mathrm{m}$ & $\mathrm{N}$ & 1760 & $1+$ & $2+$ & $2+$ & - & NA & 1 & AWD \\
\hline 24 & PMF & $48 / \mathrm{f}$ & $\mathrm{N}$ & 2900 & $2+(\mathrm{IM})$ & $2+$ & $1+$ & - & 112 & 20 & AWD \\
\hline
\end{tabular}

Abbreviations: PV, polycythemia vera; PMF, primary myelofibrosis; MPN-U, myeloproliferative neoplasm-unclassified; m, male; f, female; D, diffuse; M, mixed; N, nodular; EMHP, extramedullary hematopoietic proliferation; IM, $\geqslant 20 \%$ immature cells; AML, acute myeloid leukemia; DOD, dead-cause of disease; AWD, alive with disease; NA, not available.

Semiquantitative evaluation of hematopoietic lineages $3+>50 \%$ of total EMHP, $2+25-50 \%$ of total EMHP, $1+<25 \%$ of total EMHP.

${ }^{\mathrm{a}}$ The spleen in these two cases showed sclerosing extramedullary myeloid tumor. 

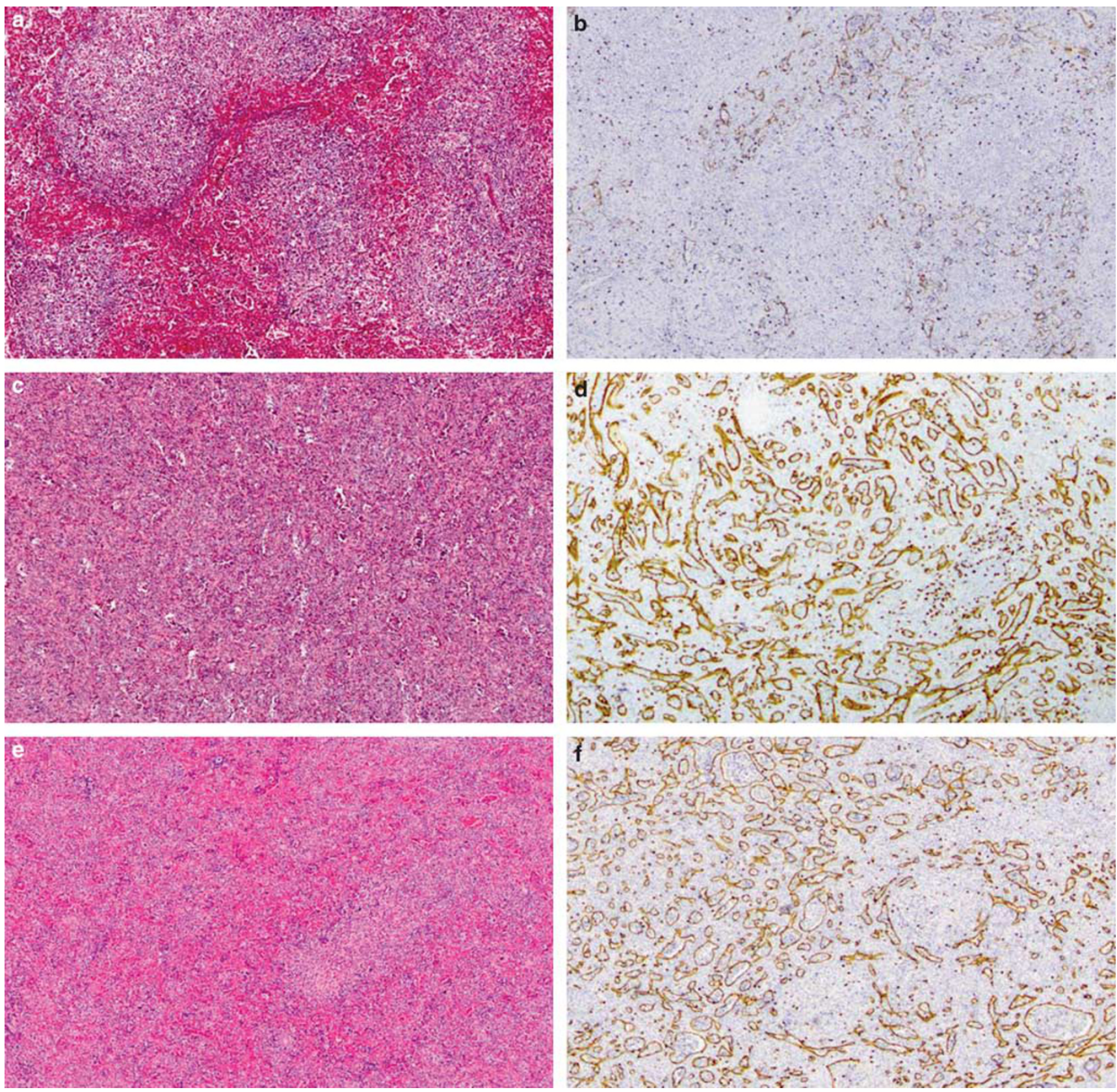

Figure 1 Histological growth patterns of splenic extramedullary hematopoietic proliferation (EMHP): nodular EMHP (a) with lack of CD8 + sinusoids (b); diffuse EMHP (c) with preservation of CD8 + sinusoids (d); and mixed EMHP (e) with partial loss of CD8 + sinusoids in the nodular areas $(\mathbf{f}$; original magnification $\times 40)$.

blood showed $15 \%$ circulating blasts and the bone marrow exhibited $40 \%$ blasts. The other two cases did not show circulating blasts in the peripheral blood. A bone marrow sample available from one of these two cases did not show increased blasts. In four of the nine cases with increased immature cells, the latter were of erythroid lineage, and in the remaining five, they were granulocytic precursors. In only one case did the immature precursors show positivity for CD34; however, in all the other cases, the immature cells were CD117 positive. Blast percentage in the peripheral blood at the time of splenectomy was available for seven of these nine patients. Bone marrow samples within 40 days before splenectomy (range 6 to 40 days, median 12 days) were available in five of these nine patients. For cases with increased immature precursors, correlation of splenic findings with the blast percentages in the peripheral blood and bone marrow samples is summarized in Table 3.

The megakaryocytic morphology was pleomorphic with a mixture of large, normal and small cells, in part showing atypical nuclear features including abnormally clumped chromatin and 

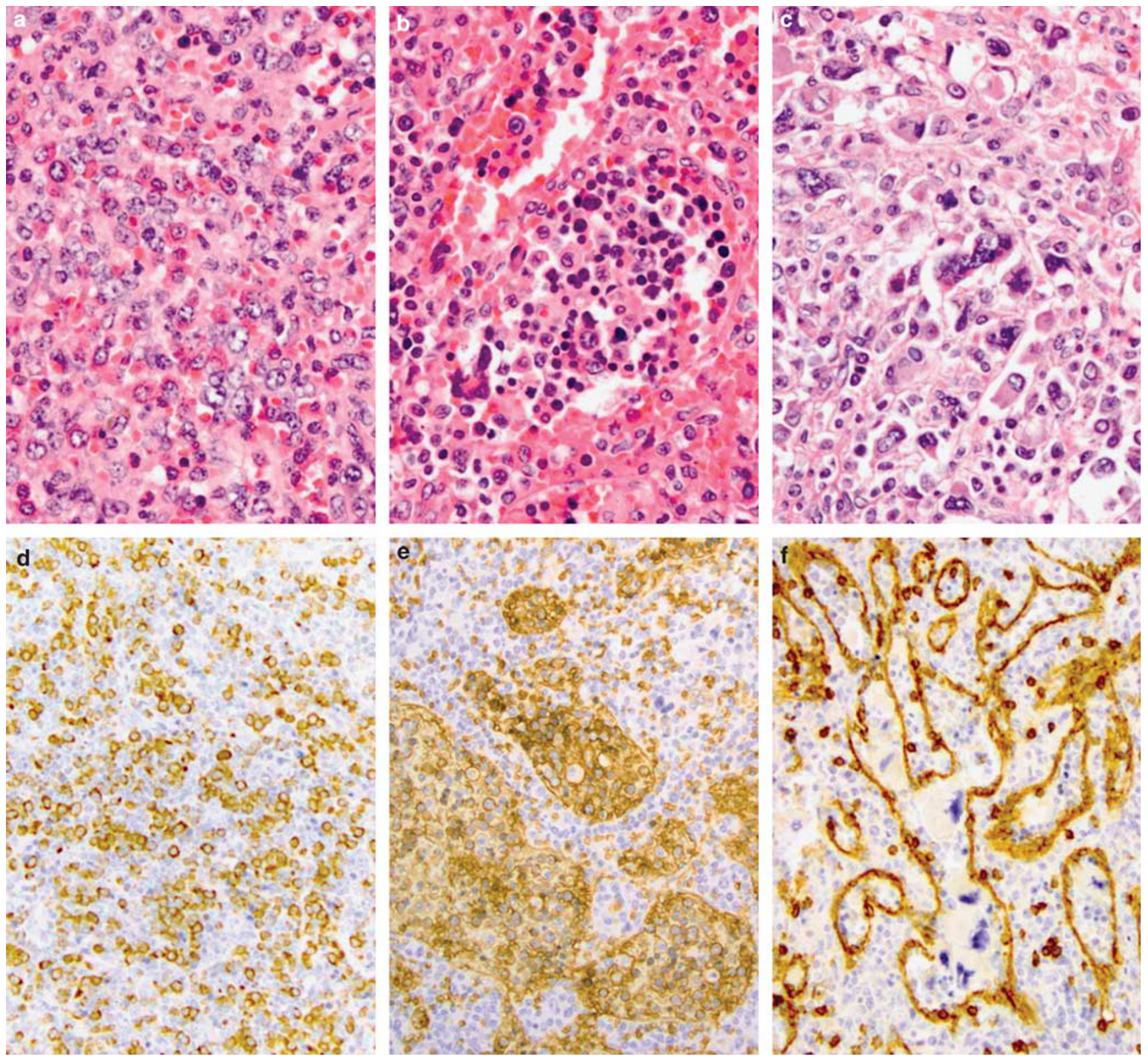

Figure 2 Spatial localization of specific hematopoietic cell lineages: granulopoiesis within splenic cords (a); erythropoiesis within sinusoids (b); and megakaryopoiesis within splenic cords (c). Myeloperoxidase-positive granulocytic cells (d); glycophorin-C-positive erythroid cells (e); and megakaryocytes within CD8 + sinusoids (f; panels (a-c) original magnification $\times 400$, (d-f) original magnification $\times 200$ ).

nuclear lobularity. Apoptotic forms were also frequently identified. In majority of the cases, the megakaryocytes were present in a scattered distribution (15 cases) with the remaining 9 cases showing additional areas of clustered megakaryocytes. The megakaryocytic number, morphology or distribution did not correlate with the specific architectural growth patterns, spleen size or degree of reticulin or trichrome fibrosis.

The architectural growth pattern, the relative proportions of each hematopoietic lineage, the presence of immature cells or megakaryocytic morphology failed to correlate with specific subtypes of myeloproliferative neoplasms.
When compared with normal spleens, those from patients with myeloproliferative neoplasms showed a variable degree of reticulin and collagen fibrosis ranging from mild to marked as identified with reticulin and trichrome stains, respectively. The degree of fibrosis was greater in nodular areas of EMHP as compared with the diffuse areas (Figure 4). The splenic sinusoidal architecture as identified by immunohistochemical staining for CD8 showed changes that paralleled the architectural growth patterns of EMHP. The CD8 + sinusoidal vasculature was preserved in the diffuse areas, whereas nodular areas of EMHP showed lack of CD8+ sinusoids (Figure 1). Immunohistochemical stains 

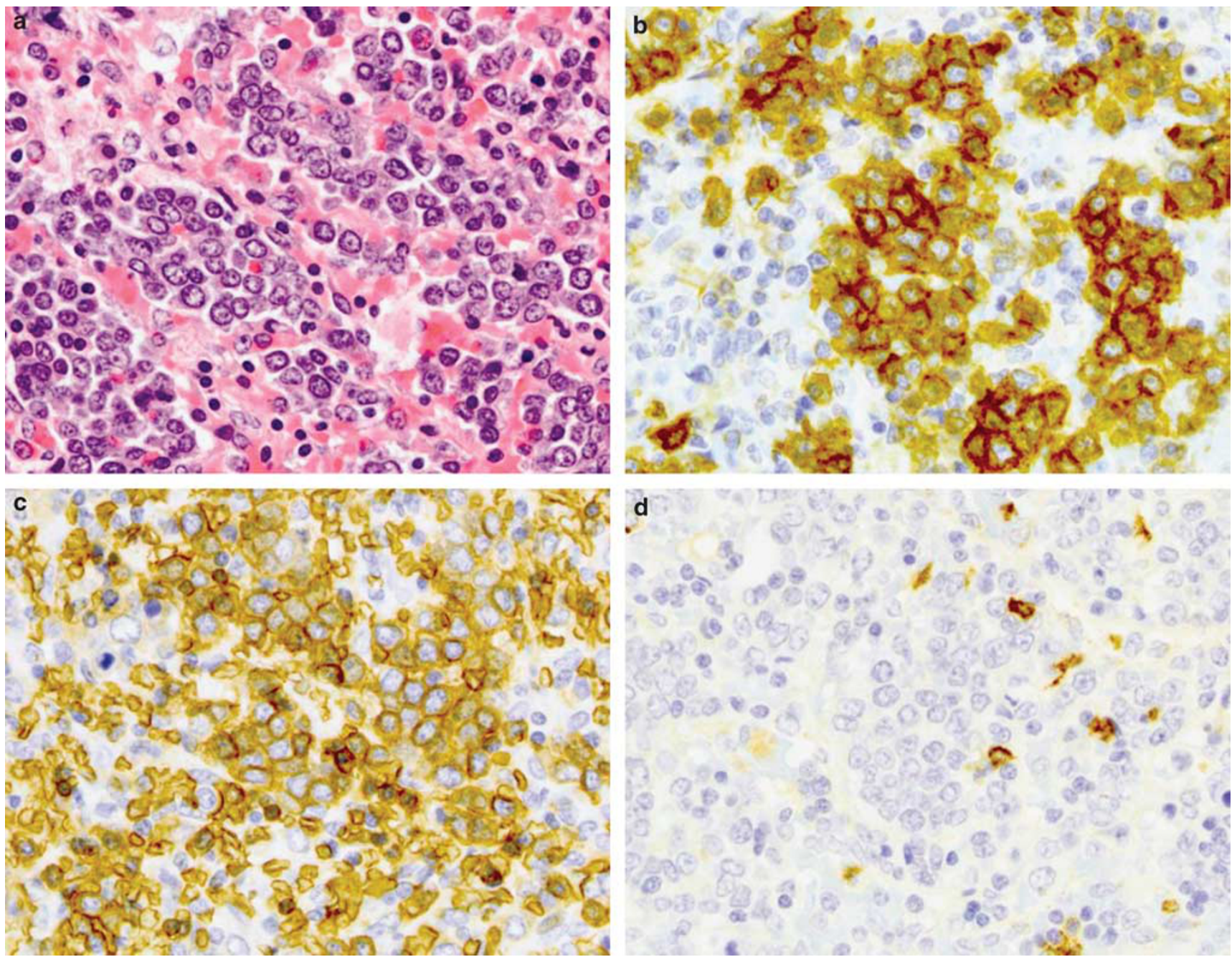

Figure 3 Immature erythroid precursors in spleen (a). The immature cells are positive for CD117 (b), glycophorin-C (c) and negative for myeloperoxidase (d; original magnification $\times 400)$.

Table 3 Correlation of findings in cases of splenic EMHP with increased immature cells with blast counts in the concurrent peripheral blood and corresponding bone marrow within 40 days before splenectomy

\begin{tabular}{lclllcr}
\hline $\begin{array}{l}\text { Serial } \\
\text { number }\end{array}$ & $\begin{array}{c}\text { Case } \\
\text { number; }\end{array}$ & $\begin{array}{c}\text { Primary } \\
\text { diagnosis }\end{array}$ & $\begin{array}{l}\text { Lineage of } \\
\text { immature cells } \\
\text { in spleen }\end{array}$ & $\begin{array}{l}\text { Histological } \\
\text { growth pattern } \\
\text { of EMHP }\end{array}$ & $\begin{array}{l}\text { Blast percentage in } \\
\text { peripheral blood at } \\
\text { time of splenectomy }\end{array}$ & $\begin{array}{c}\text { Blast percentage in bone marrow } \\
\text { sample within } \\
\text { before days }\end{array}$ \\
\hline 1 & 1 & PV & Granulocytic (AML) & Diffuse & 0 & $<5$ \\
2 & 2 & PV & Granulocytic & Diffuse & 3 & NA \\
3 & 5 & PV & Erythroid & Diffuse & 3 & NA \\
4 & 7 & PMF & Erythroid & Diffuse & NA & 40 \\
5 & 9 & PV & Erythroid & Diffuse & 15 & NA \\
6 & 13 & PMF & Erythroid & Mixed & 0 & $<5$ \\
7 & 14 & PMF & Granulocytic & Mixed & 0 & $<$ \\
8 & 22 & PV & Granulocytic & Nodular & NA & $<5$ \\
9 & 24 & PMF & Granulocytic & Nodular & NA & $<$
\end{tabular}

Abbreviations: EMHP, extramedullary hematopoietic proliferation; AML, acute myeloid leukemia; NA, not available.

The case numbers correspond to the case numbers in Table 2.

for SMA and NGFR showed loss of pericytes and adventitial reticulum cells within the nodules of EMHP. However, these structures were retained in the diffuse areas (Figure 5).
$J A K 2^{V 617 F}$ mutational analysis was informative in 14 of the 24 spleens. Mutated JAK2 V617F was identified in 7 of 14 cases, which included 4 of 8 cases with a diffuse growth pattern (3 heterozygous, 1 

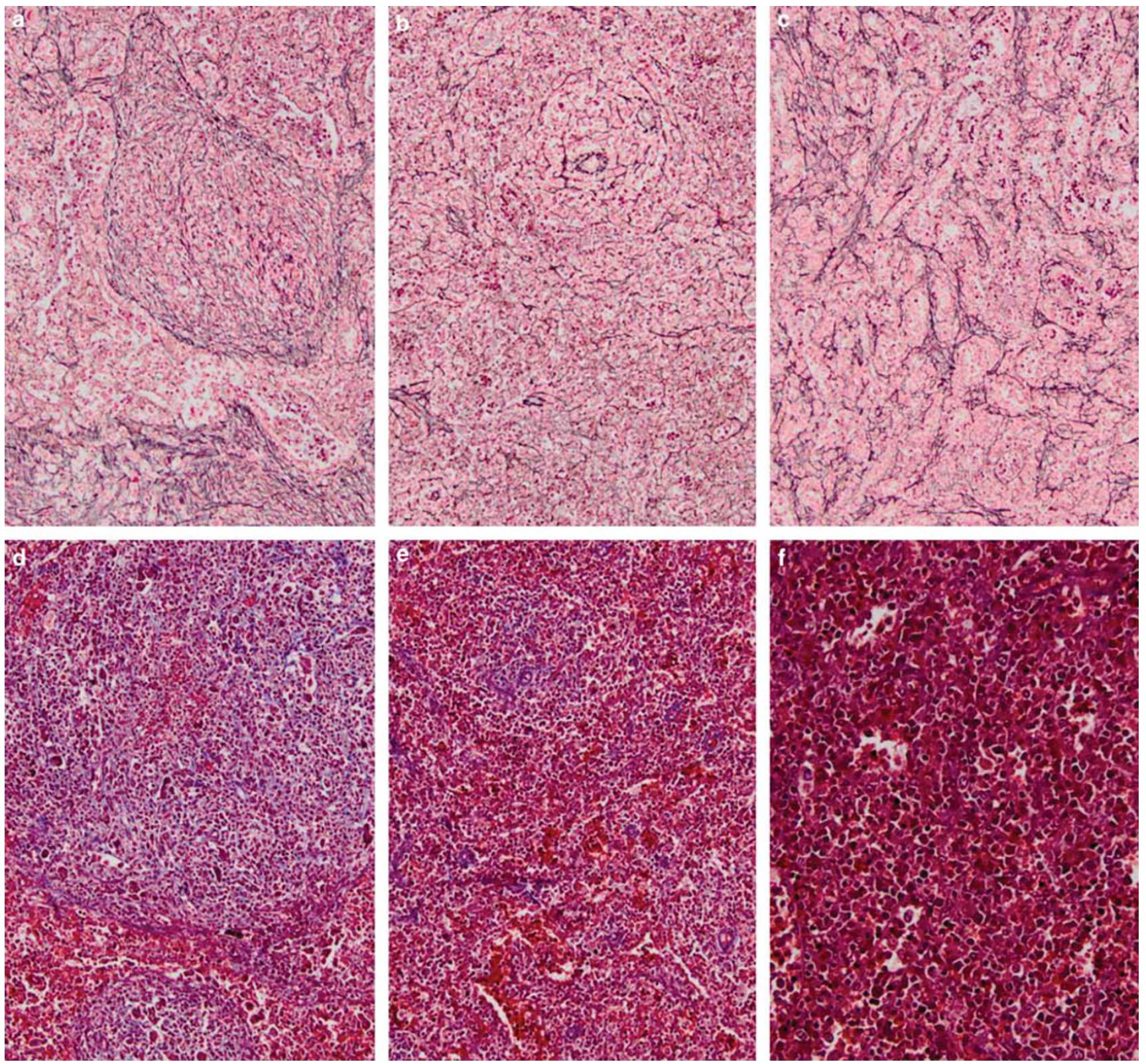

Figure 4 Reticulin and trichrome stains showing marked reticulin (a) and collagen (d) fibrosis in nodular-extramedullary hematopoietic proliferation (EMHP); greater degree of reticulin (b) and collagen (e) fibrosis within the nodular as compared with diffuse areas of mixed EMHP; and diffuse reticulin (c) and minimal collagen fibrosis (f) in diffuse EMHP (a-e: original magnification $\times 100$, $(\mathbf{f})$ original magnification $\times 200$ ).

homozygous), 1 of 3 cases with a nodular growth pattern (homozygous), and 2 of 3 cases with a mixed-nodular and diffuse growth pattern (both homozygous). When analyzed according to the subtype of Philadelphia chromosome-negative myeloproliferative neoplasm, JAK2 $2^{V 617 F}$ mutation was identified in four of six cases of polycythemia vera ( 3 homozygous, 1 heterozygous), three of seven cases of primary myelofibrosis (1 homozygous, 2 heterozygous) and was absent in the single case of myeloproliferative neoplasm unclassified. Of the nine cases with increased immature hematopoietic cells in the spleen, JAK2 ${ }^{V 617 F}$ mutational analysis was informative in five cases: two cases showed homozygous mutations (case 1 and case 24 ), one case showed heterozygous mutation (case 5 ) and two cases showed wild-type alleles (case 2 and case 22). Although the percentage of cases with a homozygous JAK2 ${ }^{V 617 F}$ mutation in the spleen appears to be much higher in polycythemia vera, the number of cases in each group is very small to infer a relationship between the $J A K 2^{V 617 F}$ mutational status and histological growth pattern, presence or absence of increased immature cells or with the subtype of the myeloproliferative neoplasm. 

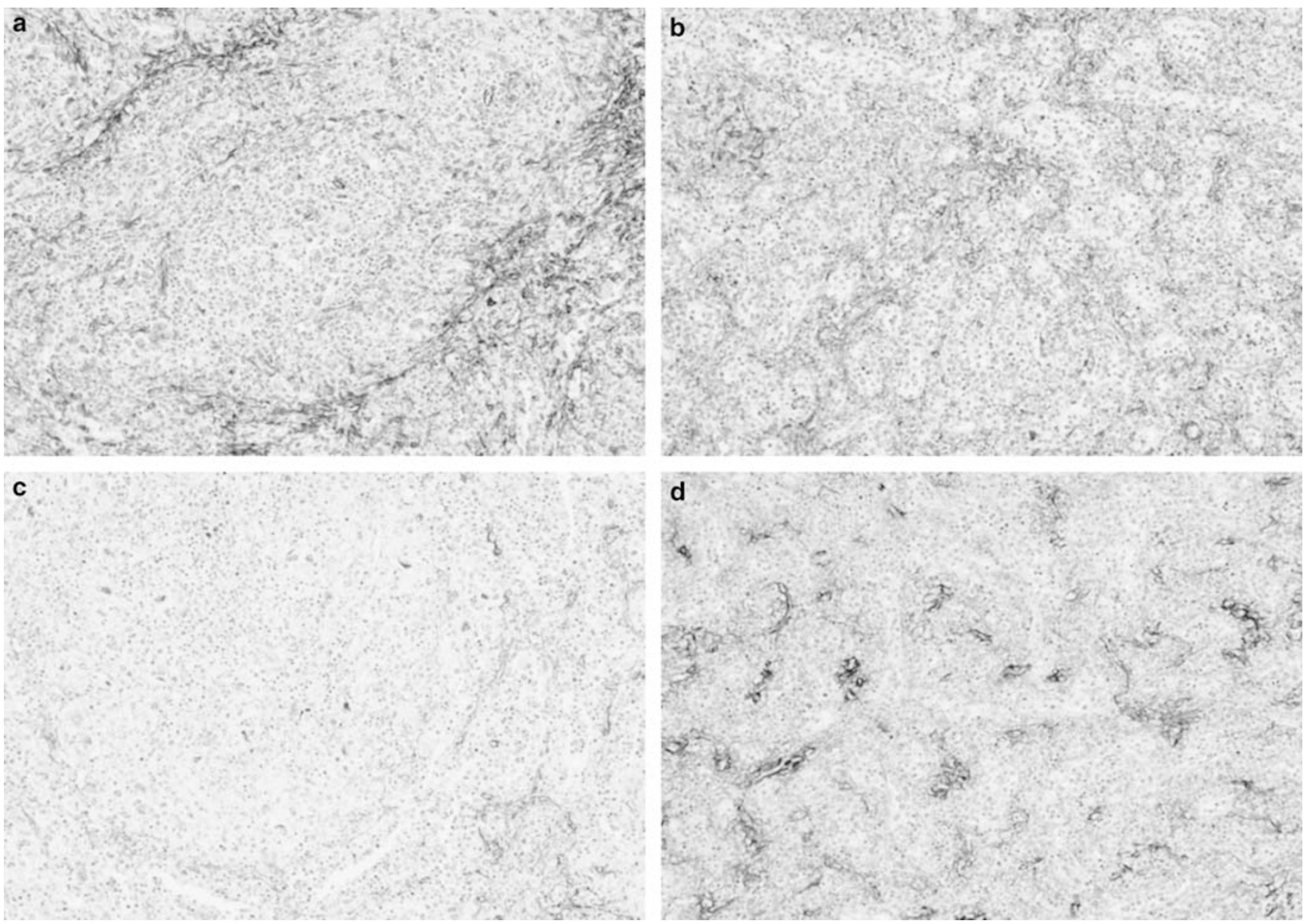

Figure 5 Pericytes and adventitial reticulum cells in the different histological growth patterns of extramedullary hematopoietic proliferation (EMHP). Loss of smooth muscle actin (SMA)-positive pericytes (a) as well as low affinity nerve growth factor (NGFR)positive adventitial reticulum cells (c) in nodular EMHP; preservation of SMA-positive pericytes (b) and NGFR-positive adventitial reticulum cells $(\mathbf{d})$ in diffuse EMHP (original magnification $\times 100$ ).

\section{Clinical Follow-Up}

Clinical follow-up was available in all 24 patients and ranged from 1 to 312 months (mean 80 months, median 67.5 months). Survival information was obtained from the clinical records as well as from the death registry. A total of 15 of the 24 patients were dead because of disease. The mortality rate did not show a correlation with the specific Philadelphia chromosome-negative myeloproliferative neoplasm subtypes: $36 \%$ in primary myelofibrosis (5 of 14 patients), $43 \%$ in polycythemia vera (3 of 7 patients) and $66.6 \%$ in myeloproliferative neoplasm-unclassifiable ( 2 of 3 patients). The duration from initial diagnosis to splenectomy was shorter in primary myelofibrosis (mean 49 months, median 31 months) as compared with polycythemia vera (mean 158 months, median 213 months). One case of polycythemia vera had a much shorter interval from initial diagnosis to splenectomy (10 months, case 5) as compared with the other cases of polycythemia vera; however, the diagnosis was confirmed based on the elevated hematocrit and red cell mass and low erythropoietin levels. Among the patients dead because of disease, the duration of disease from initial diagnosis to death was much shorter in patients with primary myelofibrosis (mean 57.8 months, median 40 months) as compared with those with polycythemia vera (mean 112 months, median 98.5 months). For the group of patients dead because of disease, the duration from diagnosis to splenectomy (mean 59.5 months, median 31 months) was shorter than the group that was alive with disease (mean 143 months, median 112 months). The mean spleen size was larger in the group dead because of disease (mean 4029g, median $3720 \mathrm{~g}$ ) than those alive with disease (mean $2484 \mathrm{~g}$, median $2200 \mathrm{~g}$ ). Information about cause of death was available in 10 cases. A total of 5 of these 10 patients died because of transformation to acute myeloid leukemia. The remaining five patients died because of septic complications secondary to decreased blood counts related to the spent phase of myeloproliferative neoplasm. In addition to the five patients that died of acute myeloid leukemia, two patients developed acute myeloid leukemia following splenectomy that responded to treatment and are alive with disease. All seven patients that 
Table 4 Summary of salient results in relation to histological growth patterns

\begin{tabular}{llccc}
\hline $\begin{array}{l}\text { Histological growth } \\
\text { pattern of EMHP }\end{array}$ & $\begin{array}{l}\text { Predominant } \\
\text { hematopoietic } \\
\text { lineage of EMHP }\end{array}$ & $\begin{array}{c}\text { Mean spleen } \\
\text { weight (g) }\end{array}$ & $\begin{array}{c}\text { Mean time to } \\
\text { splenectomy } \\
\text { (months) }\end{array}$ & $\begin{array}{c}\text { Percentage } \\
\text { survival }\end{array}$ \\
\hline Diffuse (12) & Granulocytic & 3073 (median 2517) & 83 & $33.3 \%(4 / 12)$ \\
Mixed (7) & Erythroid & 4468 (median 4510) & 93 & $29 \%(2 / 7)$ \\
Nodular (5) & Trilineage & 3587 (median 2900) & 127 & $60 \%(3 / 5)$ \\
\hline
\end{tabular}

Abbreviations: EMHP, extramedullary hematopoietic proliferation; AML, acute myeloid leukemia.

developed acute myeloid leukemia following splenectomy showed greater than $20 \%$ immature precursors in the spleen.

Although the histopathological features did not correlate with specific myeloproliferative neoplasm subtypes, there appears to be a trend showing some correlation between the histological growth pattern of splenic EMHP and disease course (Table 4). The duration from initial diagnosis to splenectomy was much shorter in patients with diffuse (83 months) and mixed growth patterns (93 months) as compared with those with nodular EMHP (127 months). The mean spleen weight was greater in mixed (4468 g) as compared with that in diffuse $(3073 \mathrm{~g})$ and nodular (3587g) EMHP. The number of patients who died because of disease was highest in cases with mixed $(5 / 7,71 \%)$ followed by diffuse $(8 / 12,67 \%)$, and the lowest in nodular EMHP (2/5, 40\%). As splenectomies for advanced-stage myeloproliferative neoplasms are not frequently performed, only a limited number of spleens are available for this study precluding evaluation of statistical significance of the differences in clinical behavior between the different histological patterns. A larger number of cases need to be studied to validate the trend observed between the histological patterns and clinical behavior in this study.

\section{Discussion}

O'Malley et al ${ }^{9}$ demonstrated that 'neoplastic hematopoietic proliferations' seen in myeloid neoplasms can be distinguished from extramedullary hematopoiesis associated with reactive conditions on the basis of a combined morphological and immunohistochemical assessment. Our study also showed features similar to those described by O'Malley et $a l^{9}$ in the spleens of patients with myeloproliferative neoplasms. These included abnormalities in the cytological features of megakaryocytes, including a tendency to cluster formation in a subset of cases.

In addition, our study of EMHP associated with Philadelphia chromosome-negative myeloproliferative neoplasms identified three distinct histological architectural growth patterns, that is, diffuse, nodular, and mixed-nodular and diffuse. These histological patterns did not correlate with the specific myeloproliferative neoplasm subtypes; however, there appeared to be a trend showing some correlation between the histological growth pattern of hematopoietic proliferation and clinical outcome. Cases with mixed and diffuse growth patterns appeared to have a shorter duration from diagnosis to splenectomy, as well as a worse overall survival than did cases with a nodular growth pattern. Progression to acute myeloid leukemia post-splenectomy was similar in the diffuse and nodular EMHP groups, and none of the cases of mixed EMHP showed leukemic transformation in the spleen or post-splenectomy. Therefore, it appears that differences in the frequency of acute leukemic transformation alone do not account for the different outcomes among the three groups of patients. A previous study by Mesa et $a l^{11}$ identified the presence of diffuse, nodular and an immature granulocytic pattern of EMHP in 213 spleens from patients with myelofibrosis with myeloid metaplasia, based on histological examination of only hematoxylin and eosin-stained sections of spleen. In our study, in addition to the diffuse and nodular histological patterns, we identified a mixed-nodular and diffuse growth pattern in $29 \%$ of the cases studied. The nodules in the cases of mixed EMHP were morphologically different from those seen in nodular EMHP in that they were much smaller in size and not as sharply demarcated from the areas of diffuse EMHP. Given that there are cases of EMHP without an obvious predominance of nodular or diffuse areas, the criteria Mesa et $a{ }^{11}$ used to classify their cases into the nodular and diffuse extramedullary hematopoiesis categories is unclear. Additionally, it is unclear if the immature granulocyte predominance pattern described by Mesa et $a l^{11}$ showed a nodular or diffuse growth pattern. A more favorable clinical course and outcome in nodular EMHP suggested by our study was also seen in the study by Mesa et al. ${ }^{11}$ Given that the proportion of diffuse areas comprised greater than $40 \%$ of the total EMHP in our cases of mixed growth pattern, it is conceivable, similar to the hypothesis by Mesa et $a l,{ }^{11}$ that hematopoietic cells within nodules of EMHP may acquire additional genetic events, leading to a diffuse growth pattern and more aggressive behavior.

The possible genetic mechanisms associated with the late stages of myeloproliferative neoplasms have begun to be elucidated. Although $J A K 2^{V 617 F}$ mutation is the most commonly identified mutation in Philadelphia chromosome-negative myeloproliferative neoplasms, the role of this mutation in disease 
progression is uncertain. ${ }^{15-18}$ Although $J A K 2^{V 617 F}$ mutational analysis did not appear to correlate with the histological growth patterns or the presence or absence of increased number of immature hematopoietic cells in our study, the small number of cases with informative results precludes any definite conclusions. Beer et $a 1^{19}$ demonstrated the presence of $R U N X 1$ in acute leukemia evolving from previous myeloproliferative neoplasm in some patients. Abdel-Wahab et al, ${ }^{20}$ by studying paired samples from patients with myeloproliferative neoplasms, who later developed acute myeloid leukemia, found evidence of acquisition of mutations in TET2, a putative tumor suppressor gene, at leukemic transformation that was not present in the predominant myeloproliferative neoplasm clone. ${ }^{19}$ The presence of mutations in IDH, ASXL1, IKZF1 and LNK has been demonstrated in cases of acute leukemic transformation of myeloproliferative neoplasms. ${ }^{21,22}$ In addition, various cytogenetic abnormalities have been shown to correlate with prognosis in myeloproliferative neoplasms, that is, favorable (sole trisomy 9, deletion 20q or deletion 13q) and unfavorable (complex karyotype or sole trisomy 8). ${ }^{23-25}$ It would be of interest to determine if significant cytogenetic abnormalities associated with the nodular and diffuse areas exist. In cases of mixed EMHP, by separately studying the nodular and diffuse areas, it might be possible to determine if indeed the diffuse areas represent progression from nodular areas. Whether these or other molecular genetic or cytogenetic alterations differ in the nodular and diffuse areas remains to be determined. In the only other study on splenic EMHP in myeloproliferative neoplasms that attempted to correlate pathological features with clinical course, Mesa et $a l^{11}$ suggested that immature granulocytic predominance was associated with a worse outcome. In our study, granulocytic predominance was seen in 5 of 12 cases of diffuse, 2 of 7 cases of mixed, and none of the cases of nodular EMHP. Erythroid predominance was seen in 4 of 12 cases of diffuse EMHP, 3 of which showed greater than $20 \%$ immature erythroid precursors and eventually progressed to acute leukemic transformation in the peripheral blood post-splenectomy. Evaluation of the predominant hematopoietic lineage of splenic EMHP in the 15 patients dead because of disease, showed a predominantly erythroid lineage in 6 cases, panmyelosis in 6 cases and a granulocytic predominance in the remaining 3 cases. Of the latter three cases, only one case showed greater than $20 \%$ immature granulocytic precursors. Therefore, the presence of granulocytic predominance by itself did not appear to correlate with outcome in our study. The limited database with very few cases showing a preponderance of immature granulocytic cells precludes evaluation of the significance of immature granulocytic precursors in our study. Of our three cases that showed greater than $50 \%$ immature precursors in the spleen, two showed predomi- nantly immature erythroid precursors (cases 9 and 13) and one case showed immature granulocytic precursors including blasts. The absence of increased blasts in the simultaneously obtained marrow and peripheral blood in one of these three patients supports the previously published hypothesis that acute leukemic transformation can occur in the spleen, at least in a subset of patients with myeloproliferative neoplasms. ${ }^{10}$

Although the reason for the different architectural organization of splenic EMHP seen in myeloproliferative neoplasms remains unclear, one could consider that differences in hematopoietic cell composition and associated structures could be due to different 'niches'. The concept of hematopoietic niches, that is, specialized microenvironments that are essential for the maintenance and function of hematopoietic cells, is generally well accepted in the bone marrow. ${ }^{3}$ However, there is very little information in relation to the existence of splenic niches. As normal hematopoietic stem cell/ hematopoietic precursor cell trafficking is thought to be dependent on the integrity of both marrow osteoblastic and vascular niches, and the spleen is not normally a site of hematopoiesis in humans, it is hypothesized that a specialized hematopoietic microenvironment is established within the spleen in myeloproliferative neoplasms to which hematopoietic stem cell/hematopoietic precursor cell from the marrow can home and engraft, leading to sustained malignant multilineage hematopoiesis and progressive splenomegaly. Migliaccio et $a{ }^{26}$ demonstrated abnormalities in the SDF-1/CXCR4 axis in the marrows of primary myelofibrosis patients that may contribute to the increased stem/progenitor cell trafficking observed in these patients. Studies using mouse models have shown that X-linked Gata 1 (low) mutation in mice induces strain-restricted myeloproliferative disorders characterized by extramedullary hematopoiesis in the spleen and liver, that Gata 1 (low) hematopoiesis is favored by the spleen as compared with the bone marrow, and that Gata 1 (low) hematopoiesis is not stem cell autonomous, but requires the permissive microenvironment of the spleen and liver. ${ }^{27}$ The presence of distinct architectural growth patterns as well as preferential spatial localization of the specific hematopoietic lineages in the spleen as found in our study, that is, erythropoiesis within vascular sinusoids, granulopoiesis within splenic cords and megakaryopoiesis within both splenic cords and vascular sinusoids, supports the possibility of distinct hematopoietic niches within the spleen. Additionally, our observation that the splenic stromal and vascular architecture is preserved in diffuse EMHP as opposed to cases with a nodular growth pattern, suggests different interactions between the neoplastic hematopoietic cells and the microenvironment in the two groups.

In summary, our study showed the presence of distinct histological growth patterns in splenic 
EMHP in Philadelphia chromosome-negative myeloproliferative neoplasms, that is, diffuse, nodular, and mixed-nodular and diffuse. These histological patterns do not correlate with the myeloproliferative neoplasm subtypes, but do appear to show some correlation with clinical behavior. Additional studies including the evaluation of molecular genetic alterations in the different histological growth patterns should be useful in elucidating the biological differences, if any, between the three groups. The overall mortality rate of $62.5 \%$ in our study further supports the view that patients with myeloproliferative neoplasms, who require splenectomy, often succumb to complications of their disease.

The specific spatial localization of each hematopoietic cell lineage suggests the possibility of different types of splenic hematopoietic 'niches'. Cytokines and chemokines are thought to have an important role in disease pathogenesis in myeloproliferative neoplasms. The source of these mediators is still not well characterized. Although megakaryocytes are thought to be a major source of these mediators, the role of the microenvironment in the production and/or response to these cytokines and chemokines remains largely elusive. Additional studies evaluating the source as well as the role of cytokines and chemokines in maintaining splenic niches are required.

\section{Acknowledgement}

This work was supported by MPD Research Consortium 2P01CA108671-05 funded by the National Cancer Institute.

\section{Disclosure/conflict of interest}

The authors declare no conflict of interest.

\section{References}

1 Thiele J, Klein H, Falk S, et al. Splenic megakaryocytopoiesis in primary (idiopathic) osteomyelofibrosis. An immunohistological and morphometric study with comparison of corresponding bone marrow features. Acta Haematol 1992;87:176-180.

2 Thiele J, Kvasnicka HM, Orazi A. Bone marrow histopathology in myeloproliferative disorders-current diagnostic approach. Semin Hematol 2005; 42:184-195.

3 Lataillade JJ, Pierre-Louis O, Hasselbalch HC, et al. Does primary myelofibrosis involve a defective stem cell niche? From concept to evidence. Blood 2008; 112:3026-3035

4 Bogani C, Ponziani V, Guglielmelli P, et al. Hypermethylation of CXCR4 promoter in CD34+ cells from patients with primary myelofibrosis. Stem Cells 2008;26:1920-1930.
5 Cho SY, Xu M, Roboz J, et al. The effect of CXCL12 processing on CD34+ cell migration in myeloproliferative neoplasms. Cancer Res 2010;70:3402-3410.

6 O’Malley DP, Orazi A, Wang M, Cheng L. Analysis of loss of heterozygosity and X chromosome inactivation in spleens with myeloproliferative disorders and acute myeloid leukemia. Mod Pathol 2005a;18: 1562-1568.

7 Konoplev S, Hsieh PP, Chang CC, et al. Janus kinase 2 $\mathrm{V} 617 \mathrm{~F}$ mutation is detectable in spleen of patients with chronic myeloproliferative diseases suggesting a malignant nature of splenic extramedullary hematopoiesis. Hum Pathol 2007;38:1760-1763.

8 Hsieh PP, Olsen RJ, O'Malley DP, et al. The role of Janus Kinase $2 \mathrm{~V} 617 \mathrm{~F}$ mutation in extramedullary hematopoiesis of the spleen in neoplastic myeloid disorders. Mod Pathol 2007;20:929-935.

9 O’Malley DP, Kim YS, Perkins SL, et al. Morphologic and immunohistochemical evaluation of splenic hematopoietic proliferations in neoplastic and benign disorders. Mod Pathol 2005b;18:1550-1561.

10 Porcu P, Neiman RS, Orazi A. Splenectomy in agnogenic myeloid metaplasia. Blood 1999;93: 2132-2134.

11 Mesa RA, Li CY, Schroeder G, Tefferi A. Clinical correlates of splenic histopathology and splenic karyotype in myelofibrosis with myeloid metaplasia. Blood 2001;97:3665-3667.

12 Chen Q, Lu P, Jones AV, et al. Amplification refractory mutation system, a highly sensitive and simple polymerase chain reaction assay, for the detection of JAK2 V617F mutation in chronic myeloproliferative disorders. J Mol Diagn 2007;9:272-276.

13 Swerdlow SH, Campo E, Harris NL, et al. (eds.) WHO Classification of Tumours of Haematopoietic and Lymphoid Tissues. IARC: Lyon, 2008.

14 Remstein ED, Kurtin PJ, Nascimento AG. Sclerosing extramedullary hematopoietic tumor in chronic myeloproliferative disorders. Am J Surg Pathol 2000; 24:51-55.

15 Levine RL, Wadleigh M, Cools J, et al. Activating mutation in the tyrosine kinase JAK2 in polycythemia vera, essential thrombocythemia, and myeloid metaplasia with myelofibrosis. Cancer Cell 2005;7: 387-397.

16 James C, Ugo V, Le Couedic JP, et al. A unique clonal JAK2 mutation leading to constitutive signalling causes polycythaemia vera. Nature 2005;434: 1144-1148.

17 Baxter EJ, Scott LM, Campbell PJ, et al. Acquired mutation of the tyrosine kinase JAK2 in human myeloproliferative disorders. Lancet 2005;365: 1054-1061.

18 Kralovics R, Passamonti F, Buser AS, et al. A gain-offunction mutation of JAK2 in myeloproliferative disorders. N Engl J Med 2005;352:1779-1790.

19 Beer PA, Delhommeau F, LeCouedic JP, et al. Two routes to leukemic transformation after a JAK2 mutation-positive myeloproliferative neoplasm. Blood 2010;115:2891-2900.

20 Abdel-Wahab O, Manshouri T, Patel J, et al. Genetic analysis of transforming events that convert chronic myeloproliferative neoplasms to leukemias. Cancer Res 2010;70:447-452.

21 Tefferi A. Novel mutations and their functional and clinical relevance in myeloproliferative neoplasms: 
JAK2, MPL, TET2, ASXL1, CBL, IDH and IKZF1. Leukemia 2010;24:1128-1138.

22 Pardanani A, Lasho T, Finke C, et al. LNK mutation studies in blast-phase myeloproliferative neoplasms, and in chronic-phase disease with TET2, IDH, JAK2 or MPL mutations. Leukemia 2010;24: 1713-1718.

23 Thoennissen NH, Krug UO, Lee DH, et al. Prevalence and prognostic impact of allelic imbalances associated with leukemic transformation of Philadelphia chromosome-negative myeloproliferative neoplasms. Blood 2010;115:2882-2890.

24 Hussein K, Pardanani AD, Van Dyke DL, et al. International Prognostic Scoring System-independent cytogenetic risk categorization in primary myelofibrosis. Blood 2010;115:496-499.

25 Tam CS, Abruzzo LV, Lin KI, et al. The role of cytogenetic abnormalities as a prognostic marker in primary myelofibrosis: applicability at the time of diagnosis and later during disease course. Blood 2009;113:4171-4178.

26 Migliaccio AR, Martelli F, Verrucci M, et al. Altered SDF-1/CXCR4 axis in patients with primary myelofibrosis and in the Gata1 low mouse model of the disease. Exp Hematol 2008;36:158-171.

27 Ghinassi B, Martelli F, Verrucci M, et al. Evidence for organ-specific stem cell microenvironments. J Cell Physiol 2010;223:460-470. 